University of Nebraska - Lincoln

DigitalCommons@University of Nebraska - Lincoln

$10-2011$

\title{
Analytic energy gradients in combined second order Møller- Plesset perturbation theory and conductorlike polarizable continuum model calculation
}

Dejun Si

University of Nebraska-Lincoln

Hui Li

University of Nebraska - Lincoln, hli4@unl.edu

Follow this and additional works at: https://digitalcommons.unl.edu/chemistryli

Part of the Chemistry Commons

$\mathrm{Si}$, Dejun and Li, Hui, "Analytic energy gradients in combined second order Møller-Plesset perturbation theory and conductorlike polarizable continuum model calculation" (2011). Hui Li Publications. 2. https://digitalcommons.unl.edu/chemistryli/2

This Article is brought to you for free and open access by the Published Research - Department of Chemistry at DigitalCommons@University of Nebraska - Lincoln. It has been accepted for inclusion in Hui Li Publications by an authorized administrator of DigitalCommons@University of Nebraska - Lincoln. 


\title{
Analytic energy gradients in combined second order Møller-Plesset perturbation theory and conductorlike polarizable continuum model calculation
}

\author{
Dejun Si and Hui $\mathrm{Li}^{\mathrm{a}}$ \\ Department of Chemistry, University of Nebraska-Lincoln, Lincoln, Nebraska 68588, USA
}

(Received 19 July 2011; accepted 20 September 2011; published online 12 October 2011)

\begin{abstract}
The analytic energy gradients in combined second order Møller-Plesset perturbation theory and conductorlike polarizable continuum model calculations are derived and implemented for spin-restricted closed shell (RMP2), Z-averaged spin-restricted open shell (ZAPT2), and spin-unrestricted open shell (UMP2) cases. Using these methods, the geometries of the $S_{0}$ ground state and the $T_{1}$ state of three nucleobase pairs (guanine-cytosine, adenine-thymine, and adenine-uracil) in the gas phase and aqueous solution phase are optimized. It is found that in both the gas phase and the aqueous solution phase the hydrogen bonds in the $\mathrm{T}_{1}$ state pairs are weakened by $\sim 1 \mathrm{kcal} / \mathrm{mol}$ as compared to those in the $\mathrm{S}_{0}$ state pairs. @ 2011 American Institute of Physics. [doi:10.1063/1.3649947]
\end{abstract}

\section{INTRODUCTION}

Dielectric solvation models ${ }^{1}$ are often used to estimate the average solvent effect on molecular structures and properties in solutions. The polarizable continuum models (the earlier DPCM (Ref. 2) and the more recent IEFPCM (Ref. 3)), conductorlike screening models (COSMO (Ref. 4) and GCOSMO (Ref. 5) or CPCM (Ref. 6)), SS(V)PE (Ref. 7) models and SM $x$ models ${ }^{8}$ are representative continuum solvation models. The conductorlike polarizable continuum model (CPCM) can be considered either as a high-dielectric limit of the more general IEFPCM or a specific version of COSMO. Recently, we derived and implemented rigorous analytic CPCM gradient for Hartree-Fock (HF), density functional theory (DFT), multiconfiguration selfconsistent-field (MCSCF), generalized valence bond theory (GVB), and time-dependent DFT (TDDFT) methods. $.{ }^{9}, 10$

In this work, we focus on second order Møller-Plesset perturbation theory (MP2) methods, including the spinrestricted closed shell RMP2, spin-unrestricted open shell UMP2, and the Z-averaged spin-restricted open shell ZAPT2 methods. ${ }^{11,12}$ The MP2 methods are accurate quantum chemical methods for recovering electron correlation energy. Except for a few cases such as aromatic ring stacking, large basis set MP2 calculation is able to give very accurate structures and intermolecular interaction energies for molecules and clusters consisting of $\mathrm{H}, \mathrm{C}, \mathrm{N}$, and $\mathrm{O}$ atoms. Pople $e t a l$. derived and implemented the MP2 analytic gradient. ${ }^{13}$ Handy and Schaefer formulated the Z-vector method so MP2 gradient and some other molecular properties can be evaluated efficiently. ${ }^{14}$ The ZAPT2 analytic gradient was derived and implemented by Fletcher et al. ${ }^{15}$ and Aikens and coworkers. ${ }^{16,17}$

Cammi et al. ${ }^{18}$ established a closed shell RMP2-PCM method that uses RHF-PCM orbitals and their energies to obtain the second order energy correction $\mathrm{E}^{(2)}$. They also derived

\footnotetext{
a) Author to whom correspondence should be addressed. Electronic mail: hli4@unl.edu.
}

and implemented a Z-vector method to determine the RMP2PCM relaxed density and analytic gradient. To the best of our knowledge, PCM methods have not been combined with open shell MP2 methods such as ZAPT2 and UMP2. In this paper, an extension of Cammi et al.'s RMP2-PCM method to ZAPT2 and UMP2 is described. These methods are used to study the $S_{0}$ and $T_{1}$ states of acetone, nucleobases, and nucleobase pairs.

\section{THEORY}

\section{A. MP2-CPCM gradient}

The RMP2-PCM method established by Cammi et al. ${ }^{18}$ can be generalized to UMP2 and ZAPT2 methods such that the corresponding restricted Hartree-Fock (RHF)-PCM, unrestricted Hartree-Fock (UHF)-PCM, and restricted open Hartree-Fock (ROHF)-PCM orbitals and orbital energies are used to obtain the second order energy correction $\mathrm{E}^{(2)}$, which is added to the corresponding HF-PCM energy.

Here, the RMP2-CPCM, UMP2-CPCM, and ZAPT2CPCM methods are considered. For all of these three methods, the first derivative (gradient) of the second order energy correction $\mathrm{E}^{(2)}$ with respect to a coordinate $x$ can be written in density matrices and basis function integrals, ${ }^{19}$

$$
\begin{aligned}
E^{(2), x}= & -\sum_{\mu \nu} W_{\mu \nu}^{(2)} S_{\mu \nu}^{x}+\sum_{\mu \nu} P_{\mu \nu}^{(2)} h_{\mu \nu}^{x} \\
& +\sum_{\mu \nu \rho \sigma} P_{\mu \nu}^{(2)} P_{\rho \sigma}\langle\mu \rho|| \nu \sigma\rangle^{x}+\sum_{\mu \nu \rho \sigma} \Gamma_{\mu \nu \rho \sigma}^{\mathrm{NS}}\langle\mu \rho|| \nu \sigma\rangle^{x} \\
& +\sum_{\mu \nu} P_{\mu \nu}^{(2)}\left(\mathbf{V}_{\mu \nu}^{T} \mathbf{q}_{N}\right)^{x}+\sum_{\mu \nu \rho \sigma} P_{\mu \nu}^{(2)} P_{\rho \sigma}\left(\mathbf{V}_{\mu \nu}^{T} \mathbf{q}_{\rho \sigma}\right)^{x}
\end{aligned}
$$

where $\mu, \nu, \rho$, and $\sigma$ are spin-orbital basis functions; $\langle\mu \rho \| \nu \sigma\rangle$ is the standard antisymmetrized two-electron integrals in the $\langle 12 \| 12\rangle$ notation; $\mathbf{h}$ is the one-electron integral; $\mathbf{S}$ is the overlap integral; $\mathbf{W}^{(2)}$ is the MP2 correction to the HF 
energy-weighted density matrix; $\mathbf{P}$ is the HF density matrix; $\mathbf{P}^{(2)}$ is the MP2 correction to the HF density matrix, which can be determined via a modified Z-vector method (to be discussed below); $\Gamma_{\mu \nu \rho \sigma}^{\mathrm{NS}}$ is the nonseparable two-particle density matrix. Here, $\mathbf{V}$ is a set (vector) of electrostatic potentials at the molecular surface points created by the electron density of the basis set product; the superscript $\mathrm{T}$ denotes transpose here and hereafter; $\mathbf{q}$ is a set (vector) of induced surface charges ( $\mathbf{q}_{\mathrm{N}}$ due to nuclei and $\mathbf{q}_{\rho \sigma}$ due to electron density of the basis set product). All $\mathbf{q}$ and $\mathbf{V}$ satisfy the linear CPCM equation

$$
\mathbf{C} \cdot \mathbf{q}=-(1-1 / \varepsilon) \mathbf{V} .
$$

The elements of the matrix $\mathbf{C}$ are $\mathbf{C}_{i i}=1.07 \sqrt{4 \pi / a_{i}}$ and $\mathbf{C}_{i j}=1 /\left|\mathbf{r}_{i}-\mathbf{r}_{j}\right|$, with $a_{i}$ being the area and $\boldsymbol{r}_{i}$ being the center coordinates of surface tessera $i$. Here, $\varepsilon$ is the dielectric constant of the solvent. Scaling factors other than $(1-1 / \varepsilon)$ were also suggested. ${ }^{4,6,20}$

The first four terms in Eq. (1) have exactly the same forms as those in the regular MP2 energy gradient formula. ${ }^{14}$ The last two terms in Eq. (1) can be written as

$$
\begin{aligned}
\sum_{\mu \nu} & P_{\mu \nu}^{(2)}\left(\mathbf{V}_{\mu \nu}^{\mathrm{T}} \mathbf{q}_{\mathrm{N}}\right)^{x}+\sum_{\mu \nu} P_{\mu \nu}^{(2)} \sum_{\rho \sigma} P_{\rho \sigma}\left(\mathbf{V}_{\mu \nu}^{\mathrm{T}} \mathbf{q}_{\rho \sigma}\right)^{x} \\
= & \left(\mathbf{V}^{(2), x}\right)^{\mathrm{T}} \mathbf{q}_{\mathrm{N}}+\left(\frac{\varepsilon}{\varepsilon-1}\right)\left(\mathbf{q}^{(2)}\right)^{\mathrm{T}} \mathbf{C}^{x} \mathbf{q}_{\mathrm{N}}+\left(\mathbf{q}^{(2)}\right)^{\mathrm{T}} \mathbf{V}_{\mathrm{N}}^{x} \\
& +\left(\mathbf{V}^{(2), x}\right)^{\mathrm{T}} \mathbf{q}_{\mathrm{HF}}+\left(\frac{\varepsilon}{\varepsilon-1}\right)\left(\mathbf{q}^{(2)}\right)^{\mathrm{T}} \mathbf{C}^{x} \mathbf{q}_{\mathrm{HF}}+\left(\mathbf{q}^{(2)}\right)^{\mathrm{T}} \mathbf{V}_{\mathrm{HF}}^{x} \\
= & \left(\mathbf{V}^{(2), x}\right)^{\mathrm{T}}\left(\mathbf{q}_{\mathrm{N}}+\mathbf{q}_{\mathrm{HF}}\right)+\frac{1}{2}\left(\frac{\varepsilon}{\varepsilon-1}\right)\left(\mathbf{q}^{(2)}\right)^{\mathrm{T}} \mathbf{C}^{x}\left(\mathbf{q}_{\mathrm{N}}+\mathbf{q}_{\mathrm{HF}}\right) \\
& +\left(\mathbf{q}^{(2)}\right)^{\mathrm{T}}\left(\mathbf{V}_{\mathrm{N}}^{x}+\mathbf{V}_{\mathrm{HF}}^{x}\right),
\end{aligned}
$$

where $\mathbf{V}^{(2), x}$ is the electrostatic field created by $\mathbf{P}^{(2)}, \mathbf{q}^{(2)}$ is the CPCM surface charge induced by the electrostatic potential created by $\mathbf{P}^{(2)}$,

$$
\begin{aligned}
\mathbf{V}^{(2), x} & =\sum_{\mu \nu} P_{\mu \nu}^{(2)} \mathbf{V}_{\mu \nu}^{x}, \\
\mathbf{q}^{(2)} & =\sum_{\mu \nu} P_{\mu \nu}^{(2)} \mathbf{q}_{\mu \nu} .
\end{aligned}
$$

The first term in the final expression of Eq. (3) represents the force between $\mathbf{q}_{\mathrm{N}}+\mathbf{q}_{\mathrm{HF}}$ (surface charge induced by nuclei and HF density $\mathbf{P}$ ) and the MP2 relaxed electron density $\mathbf{P}^{(2)}$; The second term represents the force between $\mathbf{q}_{\mathrm{N}}+\mathbf{q}_{\mathrm{HF}}$ and $\mathbf{q}^{(2)}$; the third term represents the force between $\mathbf{q}^{(2)}$ and solute nuclei, and the force between $\mathbf{q}^{(2)}$ and the HF electron density $\mathbf{P}$.

The evaluation of Eq. (3) requires the derivatives of the tessera coordinates and areas with respect to molecular geometric change. In the fixed points with variable areas (FIXPVA; Ref. 9) tessellation scheme, the positions of the surface tesserae are fixed relative to their center atoms so the tessera areas are smooth functions of their distances to neighboring spheres. Therefore, smooth and rigorous analytic derivatives of the tessera positions and areas with respect to atomic coordinates can be obtained.

\section{B. Z-vector equations}

Cammi et al. showed that by taking into account induced surface charges, the Z-vector method originally developed by Handy and Schaefer ${ }^{14}$ can be used to determine the $\mathbf{P}^{(2)}$ for RMP2-PCM methods. ${ }^{18}$ In this work, we extended the Zvector method to open shell UMP2-PCM and ZAPT2-PCM methods. We follow the notations in several recent literature papers: the doubly occupied molecular orbitals are denoted as $i, j$, and $k$, the singly occupied ones are denoted as $x$ and $y$, the virtual ones are denoted as $a, b$, and $c$, general molecular orbitals (both occupied and virtual) are denoted as $p$ and $q$.

For closed shell RMP2, the occupied-occupied and virtual-virtual blocks of the $\mathbf{P}^{(2)}$ can be readily evaluated using the RMP2 excitation amplitudes. The occupied-virtual blocks of $\mathbf{P}^{(2)}$, when written as a vector (Lagrange multiplier), can be determined using the $\mathrm{Z}$-vector equation ${ }^{14}$

$$
\sum_{b j} A_{a i b j} P_{b j}^{(2)}+\left(\varepsilon_{a}-\varepsilon_{i}\right) P_{a i}^{(2)}=L_{a i} .
$$

Here $A_{a i b j}=\langle i j|| a b\rangle+\langle i b \| a j\rangle$ is the orbital Hessian matrix, $\varepsilon_{a}$ and $\varepsilon_{i}$ are the orbital energies, and $\mathbf{L}$ is the Lagrangian. $^{14}$ For RMP2-CPCM, induced charge terms should be added to the orbital Hessian matrix $\mathbf{A}$ and the Lagrangian $\mathbf{L}$, and the occupied-occupied block of the $\mathbf{W}^{(2)}$ matrix,

$$
\begin{gathered}
A_{a i b j}^{\mathrm{pcm}}=A_{a i b j}+2 \mathbf{V}_{a i}^{T} \mathbf{q}_{b j} \\
L_{a i}^{\mathrm{pcm}}=L_{a i}+\sum_{j k} P_{j k}^{(2)} \mathbf{V}_{a i}^{T} \mathbf{q}_{j k}+\sum_{b c} P_{b c}^{(2)} \mathbf{V}_{a i}^{T} \mathbf{q}_{b c} \\
W_{i j}^{(2), \mathrm{pcm}}=W_{i j}^{(2)}-\frac{1}{2} \sum_{p q} P_{p q}^{(2)} \mathbf{V}_{i j}^{T} \mathbf{q}_{p q} .
\end{gathered}
$$

For open shell UMP2, two associated Z-vector equations for $\alpha$ and $\beta$ orbitals should be solved (see Eqs. (99) and (100) in Ref. 17). For UMP2-CPCM, induced charge terms should be added to the orbital Hessian matrix A (see Eqs. (91)-(94), (99), and (100) in Ref. 17), the Lagrangian $\mathbf{L}$ (see Eq. (98) in Ref. 17), and the occupied-occupied block of the $\mathbf{W}^{(2)}$ matrix (see Eq. (96) in Ref. 17),

$$
\begin{gathered}
\mathbf{A}_{a^{\alpha} i^{\alpha} b^{\alpha} j^{\alpha}}^{\mathrm{pcm}}=\mathbf{A}_{a^{\alpha} i^{\alpha} b^{\alpha} j^{\alpha}}+2 \mathbf{V}_{b^{\alpha} j^{\alpha}}^{T} \mathbf{q}_{a^{\alpha} i^{\alpha}} \\
\mathbf{A}_{a^{\alpha} i^{\alpha} b^{\beta} j^{\beta}}^{\mathrm{pcm}}=\mathbf{A}_{a^{\alpha} i^{\alpha} b^{\beta} j^{\beta}}+2 \mathbf{V}_{b^{\beta} j^{\beta}}^{T} \mathbf{q}_{a^{\alpha} i^{\alpha}} \\
L_{a^{\alpha} i^{\alpha}}^{\mathrm{pcm}}=L_{a^{\alpha} i^{\alpha}}+2 \sum_{j^{\alpha} k^{\alpha}} P_{j^{\alpha} k^{\alpha}}^{(2)} \mathbf{V}_{a^{\alpha} i^{\alpha}}^{T} \mathbf{q}_{j^{\alpha} k^{\alpha}} \\
+2 \sum_{j^{\beta} k^{\beta}} P_{j^{\beta} k^{\beta}}^{(2)} \mathbf{V}_{a^{\alpha} i^{\alpha}}^{T} \mathbf{q}_{j^{\beta} k^{\beta}}+2 \sum_{b^{\alpha} c^{\alpha}} P_{b^{\alpha} c^{\alpha}}^{(2)} \mathbf{V}_{a^{\alpha} i^{\alpha}}^{T} \mathbf{q}_{b^{\alpha} c^{\alpha}} \\
+2 \sum_{b^{\beta} c^{\beta}} P_{b^{\beta} c^{\beta}}^{(2)} \mathbf{V}_{a^{\alpha} i^{\alpha}}^{T} \mathbf{q}_{b^{\beta} c^{\beta}}, \\
W_{i^{\alpha} j^{\alpha}}^{(2), \mathrm{pcm}}=W_{i^{\alpha} j^{\alpha}}^{(2)}-\sum_{p^{\alpha} q^{\alpha}} P_{p^{\alpha} q^{\alpha}}^{(2)}\left(\mathbf{V}_{p^{\alpha} q^{\alpha}}^{T} \mathbf{q}_{i^{\alpha} j^{\alpha}}\right) \\
-\sum_{p^{\beta} q^{\beta}} P_{p^{\beta} q^{\beta}}^{(2)}\left(\mathbf{V}_{p^{\beta} q^{\beta}}^{T} \mathbf{q}_{i^{\alpha} j^{\alpha}}\right)
\end{gathered}
$$


Here, only the $\alpha$ equations are given. The $\beta$ equations can be obtained by switching $\alpha$ and $\beta$.

For open shell ZAPT2, a single Z-vector equation can be constructed using a nine-block (but only six independent blocks) orbital Hessian matrix A for doubly occupied, singly occupied and virtual orbitals (see Figure 3 in Ref. 16),

$$
\left(\begin{array}{ccc}
\mathbf{A}_{x i, y j} & \mathbf{A}_{x i, b j} & \mathbf{A}_{x i, b y} \\
\mathbf{A}_{a i, y j} & \mathbf{A}_{a i, b j} & \mathbf{A}_{a i, b y} \\
\mathbf{A}_{a x, y j} & \mathbf{A}_{a x, b j} & \mathbf{A}_{a x, b y}
\end{array}\right) \times\left(\begin{array}{c}
P_{y j} \\
P_{b j} \\
P_{b y}
\end{array}\right)=\left(\begin{array}{c}
L_{x i} \\
L_{a i} \\
L_{a x}
\end{array}\right) .
$$

For ZAPT2-CPCM, induced charge terms should be added to the orbital Hessian matrix A (see Eq. (81) in Ref. 16), the Lagrangian $\mathbf{L}$ (see Eq. (62)-(66) in Ref. 16) and the doubledouble, double-single and single-single blocks of the $\mathbf{W}^{(2)}$ matrix (see Eq. (55)-(61) in Ref. 16),

$$
\begin{gathered}
\mathbf{A}_{x i, y j}^{\mathrm{pcc}}=\mathbf{A}_{x i, y j}+\mathbf{V}_{y j}^{T} \mathbf{q}_{x i}, \\
\mathbf{A}_{a i, y j}^{\mathrm{pcm}}=\mathbf{A}_{a i, y j}+2 \mathbf{V}_{y j}^{T} \mathbf{q}_{a i}, \\
\mathbf{A}_{a x, y j}^{\mathrm{pcm}}=\mathbf{A}_{a x, y j}+\mathbf{V}_{y j}^{T} \mathbf{q}_{a x}, \\
\mathbf{A}_{a i, b j}^{\mathrm{pcm}}=\mathbf{A}_{a i, b j}+4 \mathbf{V}_{b j}^{T} \mathbf{q}_{a i}, \\
\mathbf{A}_{a x, b j}^{\mathrm{pcm}}=\mathbf{A}_{a x, b j}+2 \mathbf{V}_{b j}^{T} \mathbf{q}_{a x}, \\
\mathbf{A}_{a x, b y}^{\mathrm{pcm}}=\mathbf{A}_{a x, b y}+\mathbf{V}_{b y}^{T} \mathbf{q}_{a x}, \\
L_{x i}^{\mathrm{pcm}}=L_{x i}+2 \sum_{j k} P_{j k}^{(2)} \mathbf{V}_{x i}^{T} \mathbf{q}_{j k}+2 \sum_{x y} P_{x y}^{(2)} \mathbf{V}_{x i}^{T} \mathbf{q}_{x y} \\
+2 \sum_{b c} P_{b c}^{(2)} \mathbf{V}_{x i}^{T} \mathbf{q}_{b c}, \\
L_{a i}^{\mathrm{pcm}}=L_{a i}+4 \sum_{j k} P_{j k}^{(2)} \mathbf{V}_{a i}^{T} \mathbf{q}_{j k}+4 \sum_{x y} P_{x y}^{(2)} \mathbf{V}_{a i}^{T} \mathbf{q}_{x y} \\
+4 \sum_{b c} P_{b c}^{(2)} \mathbf{V}_{a i}^{T} \mathbf{q}_{b c}, \\
L_{a x}^{\mathrm{pcm}}=L_{a x}+2 \sum_{j k} P_{j k}^{(2)} \mathbf{V}_{a x}^{T} \mathbf{q}_{j k}+2 \sum_{x y} P_{x y}^{(2)} \mathbf{V}_{a x}^{T} \mathbf{q}_{x y} \\
+2 \sum_{b c} P_{b c}^{(2)} \mathbf{V}_{a x}^{T} \mathbf{q}_{b c}, \\
W_{i j}^{(2), \mathrm{pcm}}=W_{i j}^{(2)}-2 \sum_{p q} P_{p q}^{(2), \mathrm{pcm}}=W_{i x}^{(2)}-\sum_{p q} P_{p q}^{(2)} \mathbf{V}_{i j}^{T} \mathbf{q}_{p q}, \\
=W_{x y}^{(2)}-\sum_{p q} P_{p q}^{(2)} \mathbf{V}_{x y}^{T} \mathbf{q}_{p q} .
\end{gathered}
$$

For frozen core MP2 calculations, corresponding modifications can be made following the expressions in Refs. 16 and 17.

\section{Solvation energy correction}

The MP2-CPCM energy is the sum of the HF-CPCM energy and the second order energy correction $\mathrm{E}^{(2)}$. The advantage of using this formulation is that the analytic gradient can be derived. The disadvantage is that the CPCM solvation en- ergy is obtained with the HF density instead of MP2 relaxed density. Here, we describe a simple method to obtain the solvation energy using the MP2 relaxed density. In the sense of perturbation theory, the solvation energy obtained using the second order relaxed density is actually at the fourth order.

The solvation energy at the HF level is defined as

$$
G_{\text {sol }}^{\mathrm{HF}}=\frac{1}{2}\left(\mathbf{V}_{\mathrm{N}}+\mathbf{V}_{\mathrm{HF}}\right)^{\mathrm{T}}\left(\mathbf{q}_{\mathrm{N}}+\mathbf{q}_{\mathrm{HF}}\right),
$$

where $\mathbf{V}_{N}$ and $\mathbf{q}_{N}$ are the potential and induced charges created by the nuclei, $\mathbf{V}_{\mathrm{HF}}$ and $\mathbf{q}_{\mathrm{HF}}$ are the potential and induced charges created by the HF electron density.

Similar to Eq. (17), a solvation energy $G_{\text {sol }}^{\mathrm{MP} 2}$ can be defined using the MP2 relaxed density,

$$
\begin{aligned}
G_{\text {sol }}^{\mathrm{MP} 2}= & \frac{1}{2}\left(\mathbf{V}_{\mathrm{N}}+\mathbf{V}_{\mathrm{HF}}+\mathbf{V}^{(2)}\right)^{\mathrm{T}}\left(\mathbf{q}_{\mathrm{N}}+\mathbf{q}_{\mathrm{HF}}+\mathbf{q}^{(2)}\right) \\
= & \frac{1}{2}\left(\mathbf{V}_{\mathrm{N}}+\mathbf{V}_{\mathrm{HF}}\right)^{\mathrm{T}}\left(\mathbf{q}_{\mathrm{N}}+\mathbf{q}_{\mathrm{HF}}\right)+\frac{1}{2}\left(\mathbf{V}_{\mathrm{N}}+\mathbf{V}_{\mathrm{HF}}\right)^{\mathrm{T}}\left(\mathbf{q}^{(2)}\right) \\
& +\frac{1}{2}\left(\mathbf{V}^{(2)}\right)^{\mathrm{T}}\left(\mathbf{q}_{\mathrm{N}}+\mathbf{q}_{\mathrm{HF}}\right)+\frac{1}{2}\left(\mathbf{V}^{(2)}\right)^{\mathrm{T}}\left(\mathbf{q}^{(2)}\right) .
\end{aligned}
$$

Here, $\mathbf{V}^{(2)}$ and $\mathbf{q}^{(2)}$ are the potential and induced charges created by $\mathbf{P}^{(2)}$. The $G_{s o l}^{\mathrm{HF}}$ defined in Eq. (17) for HF-CPCM calculation is the first term in Eq. (18). The second and third terms in Eq. (18) are equal to each other and can be combined. We can choose to use the third term (doubled). Therefore, the correction of the solvation energy due to $\mathbf{P}^{(2)}$ is

$$
\begin{aligned}
G_{\text {sol }}^{(2)} & =G_{\text {sol }}^{\mathrm{MP} 2}-G_{\text {sol }}^{\mathrm{HF}} \\
& =\left(\mathbf{V}^{(2)}\right)^{\mathrm{T}}\left(\mathbf{q}_{\mathrm{N}}+\mathbf{q}_{\mathrm{HF}}\right)+\frac{1}{2}\left(\mathbf{V}^{(2)}\right)^{\mathrm{T}}\left(\mathbf{q}^{(2)}\right) \\
& =\left(\mathbf{V}^{(2)}\right)^{\mathrm{T}}\left(\mathbf{q}_{\mathrm{N}}+\mathbf{q}_{\mathrm{HF}}+\frac{1}{2} \mathbf{q}^{(2)}\right) .
\end{aligned}
$$

It is difficult to derive the expression of the analytic nuclear gradient for $G_{s o l}^{\mathrm{MP} 2}$ or $G_{\text {sol }}^{(2)}$. Therefore, Eq. (19) is best used as a single point energy correction.

\section{IMPLEMENTATION AND COMPUTATIONAL METHODOLOGY}

The MP2-CPCM gradient code was implemented by the authors in the GAMESS (Ref. 21) package based on several gas phase MP2 programs and the CPCM code previously implemented by $\mathrm{Li}$ and Jensen ${ }^{22}$ on the basis of the IEFPCM program originally implemented by Mennucci et al. ${ }^{3,23,24}$ The MP2 programs include the serial RMP2 and UMP2 program, ${ }^{12}$ the parallel RMP2 program implemented by Fletcher et al., ${ }^{25}$ the parallel RMP2 program implemented by Ishimura et al., ${ }^{26}$ the parallel ZAPT2 program implemented by Fletcher et al. ${ }^{15}$ and Aikens et al. ${ }^{16}$ and the parallel UMP2 program implemented by Aikens and coworkers. ${ }^{17,27}$ Numerical tests using the FIXPVA (Ref. 9) tessellation scheme for CPCM show that the accuracy of the analytic gradients obtained with GAMESS default settings is typically $10^{-6}$ hartree/bohr. Tightening the default settings leads to better accuracies such as $10^{-7}$ hartree/bohr, suggesting that the implementations are correct. Using FIXPVA, the 
TABLE I. Energies (hartree) of acetone computed using the aug-cc-pVTZ basis set. The gas phase results in each case are based on the MP2-CPCM optimized geometry in the case. CPCM effect is in $\mathrm{kcal} / \mathrm{mol}$.

\begin{tabular}{|c|c|c|c|c|c|}
\hline & $\mathrm{E}^{\mathrm{HF}}$ & $\mathrm{E}^{(2)}$ & $\mathrm{E}^{\mathrm{MP} 2}$ & $G_{\text {sol }}^{(2)}$ & $\mathrm{E}^{\mathrm{MP} 2}+G_{s o l}^{(2)}$ \\
\hline RMP2 gas phase & -192.035264 & -0.825358 & -192.860622 & 0 & -192.860622 \\
\hline RMP2-CPCM & -192.048094 & -0.822840 & -192.870934 & -0.002789 & -192.873724 \\
\hline CPCM effect & -8.05 & +1.58 & -6.47 & -1.75 & -8.22 \\
\hline ZAPT2 gas phase & -191.942713 & -0.769660 & -192.712373 & 0 & -192.712373 \\
\hline ZAPT2-CPCM & -191.948211 & -0.769946 & -192.718157 & +0.000527 & -192.717630 \\
\hline CPCM effect & -3.45 & -0.18 & -3.63 & +0.33 & -3.30 \\
\hline UMP2 gas phase & -191.951690 & -0.763264 & -192.714954 & 0 & -192.714954 \\
\hline UMP2-CPCM & -191.957147 & -0.763617 & -192.720765 & +0.000593 & -192.720172 \\
\hline CPCM effect & -3.42 & -0.22 & -3.65 & +0.37 & -3.27 \\
\hline
\end{tabular}

MP2-CPCM potential energy surfaces are rigorously continuous and smooth. Equation (19) was also implemented. The $\mathrm{E}^{\mathrm{MP} 2}+G_{\text {sol }}^{(2)}$ energy is printed out as "EMP2 $+\operatorname{EPCM}(2)$ " in the GAMESS output file.

In the CPCM calculations, spheres with radii of $0,2.124$, 2.016, and $1.908 \AA$ (after scaled by 1.20) were used for H, C, $\mathrm{N}$, and $\mathrm{O}$ atoms, respectively, to define the molecular cavity; no additional spheres were used. Using zero radii for $\mathrm{H}$ atoms means that they do not contribute to form the surface. The tessellation scheme FIXPVA was used with 240 initial tesserae per sphere. The induced surface charges were determined by a semi-iterative DIIS procedure ${ }^{24,28}$ with no charge renormalization. A dielectric constant $=78.39$ was used to represent water solvent. Only the CPCM electrostatic solvation energy was included in these calculations. The aug-cc-pvTZ and augcc-pVDZ basis set ${ }^{29}$ were used for acetone and nucleobases, respectively. Geometry optimization was performed in internal coordinates generated by the automatic delocalized coordinates algorithm. ${ }^{30}$ No symmetry was imposed in the geometry optimization.

The $\mathrm{T}_{1}$ excited states of acetone, cytosine, thymine, and uracil can be well described by an open shell ROHF or UHF wavefunction. The DFT methods can be used to include some electron correlation. For example, Zhang et al. used open shell DFT methods to study interaction of triplet uracil and thymine with water. ${ }^{31}$ Consequently, ZAPT2 and UMP2 can be used to describe electron correlation. Thiel et al. found that the $\mathrm{S}_{0}$ $\rightarrow \mathrm{S}_{1}$ excitations of acetone, cytosine, thymine, and uracil can be well described using coupled cluster linear response theory because they are dominated by single excitation of a single determinant RHF reference state. ${ }^{32}$ Usually, the $S_{0}$ $\rightarrow \mathrm{T}_{1}$ excitations have even higher weights of single excitation as compared to $S_{0} \rightarrow S_{1}$ excitations. Of course, the $T_{1}$ state ROHF or UHF wavefunction is not orthogonal to the $S_{0}$ state RHF wavefunction because the Fock operators are different in the RHF and ROHF (or UHF) equations. However, this should not be a severe problem if the orthogonality can be approximately satisfied. ${ }^{33}$ Compared to costly multireference perturbation theory and coupled cluster linear response theory methods, ZAPT2 and UMP2 methods are much more efficient and can be used to perform geometry optimization with analytic gradient. Compared to open shell DFT methods, ZAPT2 and UMP2 are able to predict correct intermolecular interactions. So, in many cases, using ZAPT2 or UMP2 methods for
$\mathrm{T}_{1}$ excited states is a viable option. The $\mathrm{T}_{1}$ excited states of adenine and guanine cannot be accurately described by ROHF or UHF wavefunction, however.

\section{RESULTS AND DISCUSSION}

\section{A. Acetone $\mathrm{S}_{0}$ and $\mathrm{T}_{1}$ states}

Acetone is used as a simple example to illustrate the MP2-CPCM methods.

The $\mathrm{S}_{0}$ ground state acetone optimized using the gas phase MP2 and solution phase MP2-CPCM has a trigonal planar skeleton. The $\mathrm{T}_{1}$ triplet state acetone optimized using the gas phase ZAPT2 and UMP2 and the solution phase ZAPT2CPCM and UMP2-CPCM shows a trigonal pyramidal skeleton. In general, the geometries in the gas phase and water are very similar to each other for the same electronic state. The RMP2 optimized carbonyl $\mathrm{C}=\mathrm{O}$ bond lengths are $1.215 \AA$ in the gas phase and $1.222 \AA$ in water, respectively. The $0.007 \AA$ lengthening of the $\mathrm{C}=\mathrm{O}$ bond is probably due to the CPCM stabilization of the partial negative charges on the $\mathrm{O}$ atom. The ZAPT2 optimized carbonyl $\mathrm{C}=\mathrm{O}$ bond lengths are 1.327 $\AA$ in the gas phase and $1.329 \AA$ in water. The UMP2 optimized bond lengths are $1.325 \AA$ in the gas phase and $1.327 \AA$ in water. The $0.002 \AA$ lengthening of the $\mathrm{C}=\mathrm{O}$ bond is smaller in magnitude than that for the $S_{0}$ state. The UHF spin contamination is not significant, with $\mathbf{S}^{2}=2.017$ in both the gas phase and the CPCM solution phase.

The energies calculated using HF and MP2 methods for acetone are listed in Table I. As expected, CPCM always leads to some solvation energy for the variational HF methods. This should be true for all molecules. The CPCM implicitly affects $\mathrm{E}^{(2)}$ by affecting the HF orbitals and orbital energies. The CPCM effects in $\mathrm{E}^{(2)}$ can be positive and negative, depending on how the orbitals and orbital energies are affected. Since $\mathrm{E}^{(2)}$ typically contains thousands to millions terms, it is very difficult to determine which terms are more important.

$G_{\text {sol }}^{(2)}$ can be either positive or negative. For $\mathrm{S}_{0}$ acetone, $G_{\text {sol }}^{(2)}$ is -0.0027895 hartree or $-1.75 \mathrm{kcal} / \mathrm{mol}$. We used RHF and RMP2 methods to calculate the dipole moments of gas phase acetone in $\mathrm{S}_{0}$ state optimized with the RMP2 method. The RHF and RMP2 results are 3.46 and 2.98 Debye, respectively. If only the dipole moment is considered, a larger dipole moment at the RHF level of theory should cause an 
TABLE II. Energies (hartree) of $\mathrm{S}_{0}$ and $\mathrm{T}_{1}$ state acetone in the last seven steps in MP2-CPCM/aug-cc-pVTZ geometry optimization processes on the $\mathrm{E}^{\mathrm{MP} 2}$ surface. The lowest energy in each series is in bold.

\begin{tabular}{lccrr}
\hline \hline \multirow{2}{*}{ Step } & \multicolumn{2}{c}{ RMP2-CPCM } & \multicolumn{2}{c}{ ZAPT2-CPCM } \\
\cline { 2 - 5 } & $\mathrm{E}^{\mathrm{MP} 2}$ & $\mathrm{E}^{\mathrm{MP} 2}+G_{\text {sol }}^{(2)}$ & $\mathrm{E}^{\mathrm{MP} 2}$ & \multicolumn{2}{c}{$\mathrm{E}^{\mathrm{MP} 2}+G_{\text {sol }}^{(2)}$} \\
\hline 1 & -192.8708802330 & -192.8736850812 & -192.7181239221 & -192.7176017801 \\
2 & -192.8709276239 & -192.8737193511 & -192.7181310854 & -192.7176045263 \\
3 & -192.8709309931 & -192.8737211474 & -192.7181383579 & -192.7176106079 \\
4 & -192.8709334255 & $-\mathbf{1 9 2 . 8 7 3 7 2 4 1 1 3 7}$ & -192.7181469876 & -192.7176180919 \\
5 & -192.8709339006 & -192.8737229130 & -192.7181553570 & -192.7176276519 \\
6 & -192.8709340181 & -192.8737233000 & -192.7181565504 & -192.7176292820 \\
7 & $-\mathbf{1 9 2 . 8 7 0 9 3 4 0 4 3 0}$ & -192.8737235289 & $-\mathbf{1 9 2 . 7 1 8 1 5 6 8 0 3 5}$ & $-\mathbf{1 9 2 . 7 1 7 6 2 9 8 9 3 1}$ \\
\hline \hline
\end{tabular}

overestimation of the magnitude of the CPCM solvation energy, and the $G_{s o l}^{(2)}$ should be positive. Therefore, the dipole moment along cannot be used to explain the negative $G_{\text {sol }}^{(2)}$.

The analytic gradient of $G_{\text {sol }}^{(2)}$ is not available. Geometry optimized on the potential energy surface of $\mathrm{E}^{\mathrm{MP} 2}$ [i.e., $\mathrm{E}^{\mathrm{HF}}$ $+\mathrm{E}^{(2)}$ in both the gas phase and CPCM solution phase] is not necessary the minimum point on the potential energy surface of $\mathrm{E}^{\mathrm{MP} 2}+G_{\text {sol }}^{(2)}$. Various tests show that $G_{\text {sol }}^{(2)}$ is virtually a constant near a minimum point on the potential energy surface of $E^{\mathrm{MP} 2}$, so the minimum point is virtually the minimum point on the potential energy surface of $\mathrm{E}^{\mathrm{MP} 2}+G_{\text {sol }}^{(2)}$. Table II lists the $\mathrm{E}^{\mathrm{MP} 2}$ and $\mathrm{E}^{\mathrm{MP} 2}+G_{\text {sol }}^{(2)}$ of the last seven steps in the geometry optimization process of $\mathrm{S}_{0}$ acetone with the RMP2CPCM method and that of the $\mathrm{T}_{1}$ acetone with the ZAPT2CPCM method. Clearly, $G_{\text {sol }}^{(2)}$ is very close to a constant. In the RMP2-CPCM case, the minimum on the $\mathrm{E}^{\mathrm{MP} 2}$ surface has an $\mathrm{E}^{\mathrm{MP} 2}+G_{\text {sol }}^{(2)}$ energy that is $5.8 \times 10^{-7}$ hartree $(3.7$ $\times 10^{-4} \mathrm{kcal} / \mathrm{mol}$ ) higher than the lowest $\mathrm{E}^{\mathrm{MP} 2}+G_{\text {sol }}^{(2)}$ energy encountered in the optimization route. In the ZAPT2-CPCM case, the minimum on the $\mathrm{E}^{\mathrm{MP} 2}$ surface indeed gives the lowest $\mathrm{E}^{\mathrm{MP} 2}+G_{\text {sol }}^{(2)}$ energy in the optimization route. Our experience shows that in $\sim 70 \%$ geometry optimization cases, the $\mathrm{E}^{\mathrm{MP} 2}$ minima coincide with the $\mathrm{E}^{\mathrm{MP} 2}+G_{\text {sol }}^{(2)}$ lowest energies. For the rest cases, the $\mathrm{E}^{\mathrm{MP} 2}+G_{\text {sol }}^{(2)}$ energies at the $\mathrm{E}^{\mathrm{MP} 2}$ minima are always within $10^{-5}$ hartree $(0.006 \mathrm{kcal} / \mathrm{mol})$ to the lowest $\mathrm{E}^{\mathrm{MP} 2}+G_{\text {sol }}^{(2)}$ energies.

We recommend the use of $\mathrm{E}^{\mathrm{MP} 2}$ for geometry optimization, and the use of $\mathrm{E}^{\mathrm{MP} 2}+G_{\text {sol }}^{(2)}$ for the total energy.

\section{B. Nucleobases}

The $\mathrm{S}_{0}$ ground state adenine, guanine, cytosine, thymine, and uracil all show planar skeletons after gas phase RMP2 optimization (Figure 1). Cytosine, thymine, and uracil should have $C_{s}$ symmetry (no symmetry is imposed in the geometry optimization). Adenine and guanine do not have symmetry because their amino groups prefer trigonal pyramidal geometries. In the $\mathrm{S}_{0}$ ground state, the methyl group of thymine has two $\mathrm{H}$ atoms pointing toward the neighboring carbonyl $\mathrm{O}$ atom.

After gas phase ZAPT2 and UMP2 optimization, the $\mathrm{T}_{1}$ excited states of cytosine, thymine, and uracil all adapt nonplanar geometries (Figure 1). In the $\mathrm{T}_{1}$ state, the methyl group of thymine has only one $\mathrm{H}$ atom pointing toward the neighbor- ing carbonyl $\mathrm{O}$ atom. The $\mathrm{T}_{1}$ excited states of uracil, thymine, and cytosine can all be considered as the results of $\pi \rightarrow \pi^{*}$ excitation of their $\mathrm{C}=\mathrm{C}$ double bonds, which destroys the $\mathrm{C}=\mathrm{C}$ double bonds, the aromaticity, and the planarity of these molecules. The changes in the bond lengths clearly indicate that the excitations are localized at the $\mathrm{C}=\mathrm{C}$ double bonds. In the $\mathrm{S}_{0}$ states optimized using the gas phase RMP2 method, the $\mathrm{C}=\mathrm{C}$ double bond length is $1.364,1.366$, and $1.371 \AA$, respectively, in uracil, thymine, and cytosine. In the $T_{1}$ states optimized using the gas phase ZAPT2 method, these bond lengths are $1.500,1.505$, and $1.492 \AA$, respectively. Other bond lengths change by at most $0.03 \AA$.
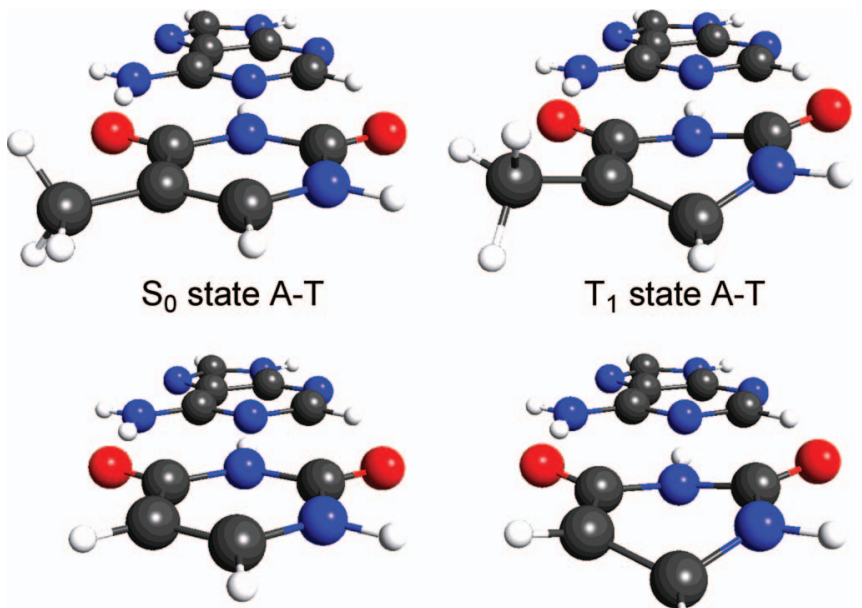

$\mathrm{S}_{0}$ state $\mathrm{A}-\mathrm{U}$

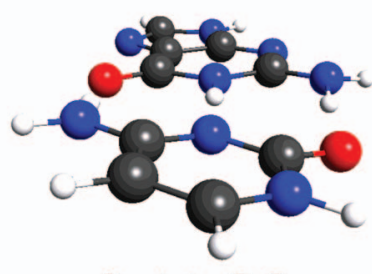

$\mathrm{S}_{0}$ state $\mathrm{G}-\mathrm{C}$

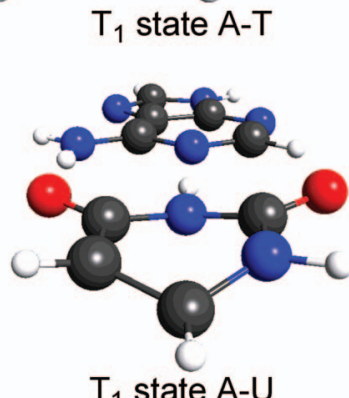

$\mathrm{T}_{1}$ state $\mathrm{A}-\mathrm{U}$

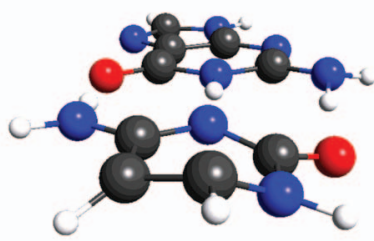

$\mathrm{T}_{1}$ state $\mathrm{G}-\mathrm{C}$

FIG. 1. Geometries of the $S_{0}$ and $T_{1}$ states of adenine-thymine (A-T), adenine-uracil (A-U), and guanine-cytosine (G-C) pairs optimized using RMP2-CPCM and ZAPT2-CPCM methods and the aug-cc-pVDZ basis set. The gas phase geometries of these pairs are similar to those in the CPCM solution phase. Free thymine, uracil, and cytosine have $S_{0}$ and $T_{1}$ geometries similar to those in the pairs. 
TABLE III. Energy (hartree) of optimized nucleobases in the gas phase $\left(\mathrm{E}^{\mathrm{MP} 2}\right)$ and solution phase $\left(\mathrm{E}^{\mathrm{MP} 2}+G_{\text {sol }}^{(2)}\right)$ using the aug-cc-pVDZ basis set. CPCM effect is in $\mathrm{kcal} / \mathrm{mol}$.

\begin{tabular}{|c|c|c|c|c|c|}
\hline & Adenine & Guanine & Cytosine & Thymine & Uracil \\
\hline RMP2 gas phase & -466.095966 & -541.177079 & -393.911022 & -452.973714 & -413.779025 \\
\hline RMP2-CPCM & -466.123432 & -541.222339 & -393.949308 & -453.004176 & -413.810450 \\
\hline CPCM effect & -17.24 & -28.40 & -24.02 & -19.12 & -19.72 \\
\hline ZAPT2 gas phase & & & -393.774560 & -452.846765 & -413.647897 \\
\hline ZAPT2-CPCM & & & -393.809807 & -452.873405 & -413.674662 \\
\hline CPCM effect & & & -22.12 & -16.72 & -16.80 \\
\hline UMP2 gas phase & & & -393.776787 & -452.846742 & -413.648774 \\
\hline UMP2-CPCM & & & -393.812780 & -452.871872 & -413.674618 \\
\hline CPCM effect & & & -22.59 & -15.77 & -16.22 \\
\hline
\end{tabular}

The solution phase RMP2-CPCM and ZAPT2-CPCM optimized $\mathrm{S}_{0}$ and $\mathrm{T}_{1}$ state geometries are very similar to those in the gas phase. The CPCM solvation can alter the bond lengths by as large as $0.01 \AA$. In the gas phase $\mathrm{S}_{0}$ and $\mathrm{T}_{1}$ states, the amino group of cytosine has a trigonal pyramidal geometry, while in the CPCM solution phases $S_{0}$ and $T_{1}$ states, it has a trigonal planar geometry.

The CPCM effects in the $\mathrm{T}_{1}$ state cytosine, thymine, and uracil are 1.91, 2.40, and $2.92 \mathrm{kcal} / \mathrm{mol}$ (ZAPT2 results in Table III) smaller in magnitude than those for the $\mathrm{S}_{0}$ states (RMP2 results in Table III). The reduction in the CPCM solvation energy is mainly caused by the changes in the electronic structure, with contributions from geometry relaxation.

The UMP2 results are very similar to the ZAPT2 results. The UHF spin contamination is not significant, with $\mathbf{S}^{2}$ being $\sim 2.05$ in all the cases.

\section{Nucleobase pairs}

The $\mathrm{S}_{0}$ ground state adenine-thymine (A-T), adenineuracil (A-U), and guanine-cytosine (G-C) hydrogen bonding pairs are optimized with the gas phase RMP2 method (Figure 1). In all of these three $S_{0}$ state pairs, the geometries of adenine, guanine, thymine, uracil, and cytosine are very similar to those of their free $\mathrm{S}_{0}$ states. The A-T pair has a planar skeleton with two $\mathrm{H}$ atoms of the thymine methyl group sticking out of the plane; the A-U pair is planar; the G-C pair is not planar because the amino group of guanine prefers a trigonal pyramidal geometry (the dihedral angle formed by the two aromatic planes is $\sim 5.5^{\circ}$ ).

The $\mathrm{T}_{1}$ excited states of A-T, A-U, and G-C pairs can also be described by ROHF, UHF, ZAPT2, and UMP2 methods because the excitations are localized in thymine, uracil, and cytosine. Here, only the ZAPT2 method is used because the UMP2 method is more costly. After the gas phase ZAPT2 optimization, the $\mathrm{T}_{1}$ state $\mathrm{A}-\mathrm{T}, \mathrm{A}-\mathrm{U}$, and G-C pairs adapt nonplanar geometries (Figure 1). In all of these three $T_{1}$ state pairs, the geometries of adenine and guanine are very similar to those of their free $\mathrm{S}_{0}$ states, and the geometries of thymine, uracil, and cytosine are very similar to those of their free $T_{1}$ states. Therefore, these $T_{1}$ excited state pairs can be considered as dimers formed by $S_{0}$ state adenine/guanine and $T_{1}$ state uracil/thymine/cytosine. In the $\mathrm{S}_{0}$ state pairs optimized using the gas phase RMP2 method the $\mathrm{C}=\mathrm{C}$ double bond lengths are $1.365,1.367$, and $1.369 \AA$, respectively, in uracil, thymine, and cytosine. In the $\mathrm{T}_{1}$ state optimized using the gas phase ZAPT2 method these bond lengths are 1.498, 1.505, and $1.492 \AA$, respectively. These values are almost identical to those in the free uracil, thymine, and cytosine. In the pairs, other covalent bond lengths change at most by $0.03 \AA$ from $S_{0}$ to $T_{1}$, while the hydrogen bond lengths change more significantly.

The geometries after solution phase RMP2-CPCM and ZAPT2-CPCM optimization are very similar to those in the gas phase. The CPCM solvation can alter covalent bond lengths by as large as $0.03 \AA$, and hydrogen bond lengths by as large as $0.14 \AA$. For example, in the $\mathrm{S}_{0}$ state A-U pair, the middle hydrogen bond is shortened by $0.10 \AA$, while the other hydrogen bond is lengthened by $0.14 \AA$, ongoing from the gas phase to CPCM.

The gas phase formation energy of the $\mathrm{S}_{0}$ ground state $\mathrm{G}$ $\mathrm{C}$ pair is $-30.27 \mathrm{kcal} / \mathrm{mol}$ as calculated from the difference between the gas phase RMP2 optimized energy of the G-C pair (Table IV) and the sum of the RMP2 optimized monomer energies (Table III). The solution phase formation energy of the $\mathrm{S}_{0}$ state $\mathrm{G}-\mathrm{C}$ pair is $-10.36 \mathrm{kcal} / \mathrm{mol}$ as calculated from the RMP2-CPCM optimized energies. So, the formation of the $\mathrm{S}_{0}$ state G-C pair in water is $19.91 \mathrm{kcal} / \mathrm{mol}$ less favorable than that in the gas phase because water tends to dissolve the pair. The gas phase formation energy of the $\mathrm{T}_{1}$ state G-C pair is $-29.86 \mathrm{kcal} / \mathrm{mol}$ as calculated from the difference between the gas phase ZAPT2 optimized energy of the G-C pair and the sum of the RMP2 optimized energy of $S_{0}$ guanine and the ZAPT2 optimized energy of $T_{1}$ cytosine. The solution phase formation energy of the $T_{1}$ state G-C pair is $-9.49 \mathrm{kcal} / \mathrm{mol}$. The values for the A-U and A-T pairs are listed in Table IV for comparison.

Clearly, in both the gas phase and the solution phase, the hydrogen bonds in the $\mathrm{T}_{1}$ state A-U, A-T, and G-C pairs is $\sim 1 \mathrm{kcal} / \mathrm{mol}$ weaker than those in the $\mathrm{S}_{0}$ state pairs, and the CPCM solvation does not appear to significantly affect the weakening of the hydrogen bonding ongoing from $S_{0}$ to $T_{1}$ state. We performed aug-cc-pVQZ (Ref. 29) interaction analysis using the localized molecular orbital energy decomposition analysis (LMO-EDA) method $^{34}$ for select pairs and found that the basis set superposition error (BSSE) in the formation energy calculated using the MP2/aug-cc-pVDZ methods is $\sim 4 \mathrm{kcal} / \mathrm{mol}$. We will publish the results of the LMO-EDA 
TABLE IV. Energy (hartree) of optimized nucleobase pairs in the gas phase $\left(\mathrm{E}^{\mathrm{MP} 2}\right)$ and solution phase $\left(\mathrm{E}^{\mathrm{MP} 2}+G_{\text {sol }}^{(2)}\right)$ using the aug-cc-pVDZ basis set. The CPCM effect and formation energy are in $\mathrm{kcal} / \mathrm{mol}$.

\begin{tabular}{|c|c|c|c|c|c|c|}
\hline & \multicolumn{2}{|c|}{ Adenine-uracil } & \multicolumn{2}{|c|}{ Adenine-thymine } & \multicolumn{2}{|c|}{ Guanine-cytosine } \\
\hline & Absolute & Formation & Absolute & Formation & Absolute & Formation \\
\hline RMP2 gas phase & -879.903366 & -17.80 & -919.097953 & -17.74 & -935.136344 & -30.27 \\
\hline RMP2-CPCM & -879.946358 & -7.83 & -919.140111 & -7.85 & -935.188155 & -10.36 \\
\hline CPCM effect & -26.98 & 9.97 & -26.45 & 9.89 & -32.51 & 19.91 \\
\hline ZAPT2 gas phase & -879.770446 & -16.68 & -918.969335 & -16.69 & -934.999236 & -29.86 \\
\hline ZAPT2-СРCM & -879.809274 & -7.01 & -919.008226 & -7.15 & -935.047277 & -9.49 \\
\hline CPCM effect & -24.36 & 9.67 & -24.41 & 9.54 & -30.15 & 20.37 \\
\hline
\end{tabular}

interaction analysis for these pairs in a separate paper. Here, we note that the BSSE do not significantly affect the relative energies discussed earlier.

The CPCM solvation effects in the $\mathrm{T}_{1}$ state G-C, A-T, and A-U pairs are $2.36,2.05$, and $2.61 \mathrm{kcal} / \mathrm{mol}$ smaller in magnitude than those for the $\mathrm{S}_{0}$ states (Table IV). These differences are similar to those $(1.91,2.40$, and $2.92 \mathrm{kcal} / \mathrm{mol})$ found for free cytosine, thymine, and uracil, implying that they are caused by local changes in the electronic structures and molecular geometries of these molecules.

\section{CONCLUSION}

Analytic energy gradient are derived and implemented for MP2-CPCM methods. Very accurate gradient and smooth and continuous potential energy surfaces are obtained by using the FIXPVA surface tessellation scheme for CPCM. All closed shell and open shell MP2 methods (RMP2, ZAPT2, and UMP2, serial and parallel programs) in GAMESS are enabled to perform MP2-CPCM gradient calculations. A better CPCM solvation energy can be obtained efficiently by using the MP2 relaxed density. The open shell ZAPT2 and ZAPT2-CPCM methods are used to study the $\mathrm{T}_{1}$ excited states of three nucleobase pairs (adenine-thymine, adenineuracil, and guanine-cytosine) in the gas phase and aqueous solution phase. It is found that in both the gas phase and solution phase, the hydrogen bonds in the $\mathrm{T}_{1}$ excited state base pairs are $\sim 1 \mathrm{kcal} / \mathrm{mol}$ weaker than those in the $S_{0}$ states.

\section{ACKNOWLEDGMENTS}

This work was supported by the USA National Science Foundation (Award No. 1010674) and start-up funds from the University of Nebraska-Lincoln.

${ }^{1}$ J. Tomasi, B. Mennucci, and R. Cammi, Chem. Rev. $105(8), 2999$ (2005).

${ }^{2}$ S. Miertus, E. Scrocco, and J. Tomasi, Chem. Phys. 55(1), 117 (1981).

${ }^{3}$ E. Cances, B. Mennucci, and J. Tomasi, J. Chem. Phys. 107(8), 3032 (1997).

${ }^{4}$ A. Klamt and G. Schuurmann, J. Chem. Soc., Perkin Trans. 2(5), 799 (1993).

${ }^{5}$ T. N. Truong and E. V. Stefanovich, Chem. Phys. Lett. 240(4), 253 (1995).

${ }^{6}$ V. Barone and M. Cossi, J. Phys. Chem. A 102(11), 1995 (1998).

${ }^{7}$ D. M. Chipman, Theor. Chem. Acc. 107(2), 80 (2002); D. M. Chipman and M. Dupuis, ibid. 107(2), 90 (2002).
${ }^{8}$ A. V. Marenich, R. M. Olson, C. P. Kelly, C. J. Cramer, and D. G. Truhlar, J. Chem. Theory Comput. 3(6), 2011 (2007).

${ }^{9}$ P. Su and H. Li, J. Chem. Phys. 130(7), 074109 (2009).

${ }^{10}$ Y. Wang and H. Li, J. Chem. Phys. 133(3), 034108 (2010).

${ }^{11}$ J. A. Pople, J. S. Binkley, and R. Seeger, Int. J. Quantum Chem. 10(S10), 1 (1976); C. Møller and M. S. Plesset, Phys. Rev. 46(7), 618 (1934); T. J. Lee and D. Jayatilaka, Chem. Phys. Lett. 201(1-4), 1 (1993); J. S. Binkley and J. A. Pople, Int. J. Quantum Chem. 9(2), 229 (1975); T. J. Lee, A. P. Rendell, K. G. Dyall, and D. Jayatilaka, J. Chem. Phys. 100(10), 7400 (1994); I. M. B. Nielsen and E. T. Seidl, J. Comput. Chem. 16(10), 1301 (1995).

${ }^{12}$ M. J. Frisch, M. Head-Gordon, and J. A. Pople, Chem. Phys. Lett. 166(3), 275 (1990).

${ }^{13}$ J. A. Pople, R. Krishnan, H. B. Schlegel, and J. S. Binkley, Int. J. Quantum Chem. 16(S13), 225 (1979).

${ }^{14}$ N. C. Handy and H. F. Schaefer III, J. Chem. Phys. 81(11), 5031 (1984).

${ }^{15}$ G. D. Fletcher, M. S. Gordon, and R. S. Bell, Theor. Chem. Acc. 107(2), 57 (2002).

${ }^{16}$ C. M. Aikens, G. D. Fletcher, M. W. Schmidt, and M. S. Gordon, J. Chem. Phys. 124(1), 014107 (2006).

${ }^{17}$ C. M. Aikens, S. P. Webb, R. L. Bell, G. D. Fletcher, M. W. Schmidt, and M. S. Gordon, Theor. Chem. Acc. 110(4), 233 (2003).

${ }^{18}$ R. Cammi, B. Mennucci, and J. Tomasi, J. Phys. Chem. A 103(45), 9100 (1999).

${ }^{19}$ J. E. Rice and R. D. Amos, Chem. Phys. Lett. 122(6), 585 (1985); E. D. Simandiras, R. D. Amos, and N. C. Handy, Chem. Phys. 114(1), 9 (1987).

${ }^{20}$ M. Cossi, N. Rega, G. Scalmani, and V. Barone, J. Comput. Chem. 24(6), 669 (2003).

${ }^{21}$ M. W. Schmidt, K. K. Baldridge, J. A. Boatz, S. T. Elbert, M. S. Gordon, J. H. Jensen, S. Koseki, N. Matsunaga, K. A. Nguyen, S. J. Su, T. L. Windus, M. Dupuis, and J. A. Montgomery, J. Comput. Chem. 14(11), 1347 (1993); M. S. Gordon and M. W. Schmidt, in Theory and Applications of Computational Chemistry, edited by C. E. Dykstra, G. Frenking, K. S. Kim, and G. E. Scuseria (Elsevier, New York, 2005).

${ }^{22} \mathrm{H}$. Li and J. H. Jensen, J. Comput. Chem. 25(12), 1449 (2004).

${ }^{23}$ J. Tomasi, B. Mennucci, and E. Cances, J. Mol. Struct.: THEOCHEM 464(1-3), 211 (1999).

${ }^{24}$ H. Li, C. S. Pomelli, and J. H. Jensen, Theor. Chem. Acc. 109(2), 71 (2003).

${ }^{25}$ G. D. Fletcher, M. W. Schmidt, and M. S. Gordon, in Advances in Chemical Physics, (Wiley, New York, 1999), Vol. 110, p. 267.

${ }^{26}$ K. Ishimura, P. Pulay, and S. Nagase, J. Comput. Chem. 27(4), 407 (2006); K. Ishimura, P. Pulay, and S. Nagase, 28(12), 2034 (2007).

${ }^{27}$ C. M. Aikens and M. S. Gordon, J. Phys. Chem. A 108(15), 3103 (2004).

${ }^{28}$ C. S. Pomelli, J. Tomasi, and V. Barone, Theor. Chem. Acc. 105(6), 446 (2001).

${ }^{29}$ T. H. Dunning, J. Chem. Phys. 90(2), 1007 (1989).

${ }^{30}$ J. Baker, A. Kessi, and B. Delley, J. Chem. Phys. 105(1), 192 (1996).

${ }^{31}$ R. b. Zhang, T. Zeegers-Huyskens, A. Ceulemans, and M. T. Nguyen, Chem. Phys. 316(1-3), 35 (2005).

${ }^{32}$ M. Schreiber, M. R. Silva-Junior, S. P. A. Sauer, and W. Thiel, J. Chem. Phys. 128(13), 134110 (2008).

${ }^{33}$ A. T. B. Gilbert, N. A. Besley, and P. M. W. Gill, J. Phys. Chem. A 112(50), 13164 (2008).

${ }^{34}$ P. Su and H. Li, J. Chem. Phys. 131(1), 014102 (2009). 